\title{
Structural observability analysis and EKF based parameter estimation of building heating models
}

\author{
D. Wathsala U. Perera M. Anushka S. Perera Carlos F. Pfeiffer Nils-Olav Skeie
}

Faculty of Technology, University College of Southeast Norway, Postboks 203, N-3901, Porsgrunn, Norway. E-mail: wathsala.perera@hit.no

\begin{abstract}
Research for enhanced energy-efficient buildings has been given much recognition in the recent years owing to their high energy consumptions. Increasing energy needs can be precisely controlled by practicing advanced controllers for building Heating, Ventilation, and Air-Conditioning (HVAC) systems. Advanced controllers require a mathematical building heating model to operate, and these models need to be accurate and computationally efficient. One main concern associated with such models is the accurate estimation of the unknown model parameters.

This paper presents the feasibility of implementing a simplified building heating model and the computation of physical parameters using an off-line approach. Structural observability analysis is conducted using graph-theoretic techniques to analyze the observability of the developed system model. Then Extended Kalman Filter (EKF) algorithm is utilized for parameter estimates using the real measurements of a single-zone building. The simulation-based results confirm that even with a simple model, the EKF follows the state variables accurately. The predicted parameters vary depending on the inputs and disturbances.
\end{abstract}

Keywords: Extended Kalman Filter, Mathematical models, Parameter estimation, Single-zone building, Structural observability

\section{Introduction}

Buildings use $40 \%$ of the primary energy supply in the world, and the same figures apply to the European Union [Perez-Lombard et al. (2008)]. Out of that, Heating, Ventilation and Air-Conditioning (HVAC) systems are responsible for the majority of the overall energy consumed in buildings. Increasing energy demands can be reduced by upgrading the relevant building components and by using advanced controllers for the operation of the HVAC systems. Advanced controllers require a building heating model to operate [Dounis and Caraiscos (2009)]. Identification of a suitable model for the control system is essential for better use of energy in buildings. The model can be used to control indoor climate, estimate building heating and cooling times, forecast energy consumption and describe the energy performance of the building. These models usually need to be accurate and computationally efficient. However, obtaining such models for buildings is difficult due to highly time-varying and non-linear nature of the weather conditions and building dynamics. Further, model-based controllers, often need information of all state variables, while not all state variables in a building model are measurable [Maasoumy et al. (2013)]. According to Maasoumy et al. (2013), it is also challenging to estimate the thermal parameters accurately.

Buildings have uncertain and time-varying heat transfer characteristics. They are profoundly dependent on the ambient weather conditions and occupant behaviours. The thermal parameters of a building such as heat transfer coefficients are highly reliant on the outside weather conditions. Owing to the continuous 
ambient changes, the mathematical model describing the dynamics of the building has uncertain parameters. Therefore, the algorithms used for evaluation of these parameters should be time-varying and hence be adaptive [Maasoumy et al. (2013)]. However, first principles models for the heating of buildings may present a rather large number of parameters. The complexity of the parameter identification depends on the complexity of the model. If the building has several zones at different temperatures, identification becomes even more challenging. Further, practical difficulties such as outdoor air exchange, energy inputs, moisture and occupant behaviours can make the system more complicated. Concurrently, the parameter identification of building models can be important for the development of simplified models, like application in Building Energy Management Systems (BEMS), optimal control, energy audits and characterization of building components.

When physical insight is used to propose a model for the building heat dynamics with differential equations, Maximum Likelihood method [Madsen and Holst (1995), Bacher and Madsen (2011)] and Maximum a posteriori estimation [Kristensen et al. (2004)] and Kalman Filtering [Maasoumy et al. (2013), Fux et al. (2014), Martincevic et al. (2014), Radecki and Hencey (2012)] have been used for parameter identification. They are called hybrid or grey box modelling approaches that blend the two extremes (white box and black box) in various degrees. Grey-box approaches were used by Walker (2005), Bacher and Madsen (2011) to estimate state variables and unknown thermal parameters for buildings.

\section{Physical models of buildings}

Physical models of dynamical systems such as buildings can be formulated with ordinary differential equations. They are typically developed by using the laws of physics such as conservation of mass and energy. Linear and non-linear dynamic models for buildings can be developed using different techniques. Non-linear models provide a better prediction of building thermal dynamics while they are computationally intensive. Linear models are obtained following the simplification of non-linear models and tend to have limited computational intensity while restrained to the operating zones they are tuned for [Maasoumy et al. (2013)]. Sometimes, it is quite tricky to assess the accuracy of the linear models owing to the inevitable idealisations and simplifications according to Madsen and Holst (1995). However, Maasoumy et al. (2013) have stated that these linear models can be adjusted by using an adaptive parameter estimation technique such that the building parameters are updated as the inside and outside environment changes. The combination is a hybrid model and an upgraded version of the linear physical model of the building. Grey-box modelling can also be applied to non-linear models simultaneously with linear models.

A mechanistic model is developed for a single-zone experimental construction built in 2014 and located at the University College of Southeast Norway in Porsgrunn, Norway. The building has an inside volume of $9.4 \mathrm{~m}^{3}$, and it is completely sealed such that no ventilation is provided from outside. A view of the building and the floor plan are presented in Figures 1 and 2.

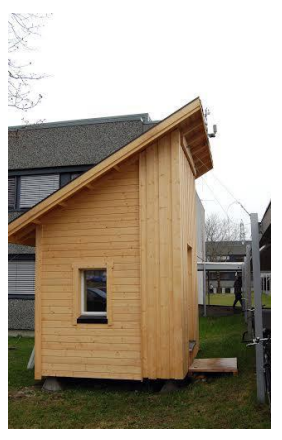

Figure 1: The experimental single-zone building from east direction.

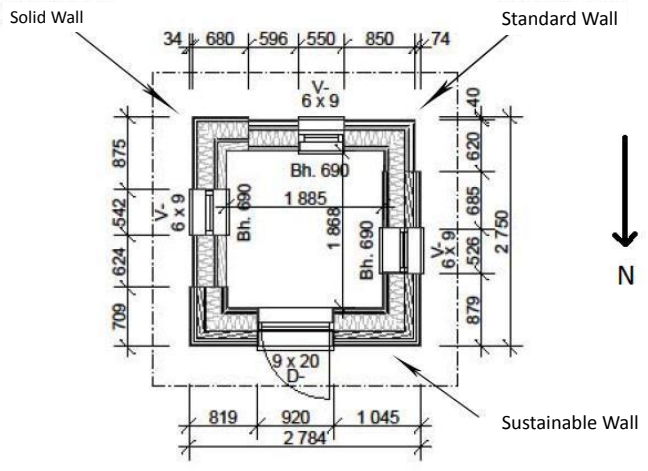

Figure 2: Plan of the test building. Measurements are in $\mathrm{mm}$.

There are three distinctive walls in the building. Each wall, roof and floor has entirely different composition based on the materials used for construction. Therefore, each component of the building envelope has separate heat transfer characteristics. The test building is constructed on support structures made out of concrete, and, therefore, the floor of the building is not in contact with the ground. Hence, all building com- 
ponents interact with outside air. The building has three windows of each $60 \times 90 \mathrm{~cm}^{2}$ into the south, west and east directions. The door is on the north wall of the building and has a dimension of $90 \times 120 \mathrm{~cm}^{2}$. An electrical heater with a maximum capacity of $370 \mathrm{~W}$ is used inside the building for heating with a thermostat controller. Also, a computer is used inside the building for data logging which contributes to use a power in between $100 \mathrm{~W}-120 \mathrm{~W}$. The interior humidity of the test building is controlled using a humidifier.

There is a measurement system inside the experimental setup which consists of a weather station and a DAQ device (NI USB-6218) connected to temperature sensors (TMP36), humidity sensors (Honeywell 4000) and a power consumption sensor. Power consumption, inside temperature, inside humidity, outside temperature, three wall temperatures, roof temperature and floor temperature are the interested measurements for this study.

A linear physical model is developed for the abovementioned test building, and it is inherited from the models presented in Perera et al. (2014a) and Perera et al. (2014b). The energy balance equation for inside air is derived and presented by the equation (1). To obtain a simple model for control purposes, we lessen the number of independent parameters by considering a common overall heat transfer coefficient $\left(\mathrm{U}_{o}\right)$ and a common thermal diffusivity $(\alpha)$ for all components of the building envelope. The symbols used in the model equations are explained in Table 2.

$$
\begin{gathered}
\frac{d T_{i}}{d t}=\frac{-M_{i} A_{t o t} U_{o}}{\rho_{i} V_{i}\left(M_{i} c_{p_{i}}-R\right)} T_{i}+\frac{M_{i}}{\rho_{i} V_{i}\left(M_{i} c_{p_{i}}-R\right)} \dot{Q}_{h} \\
+\frac{M_{i} A_{t o t} U_{o}}{\rho_{i} V_{i}\left(M_{i} c_{p_{i}}-R\right)} T_{o}
\end{gathered}
$$

The next step is to formulate the equations for three walls, roof and floor to depict their heat transfer characteristics. These equations are derived after discretizing the one-dimensional heat equation using finite difference method.

$$
\begin{gathered}
\frac{d T_{m w}}{d t}=\frac{2 \alpha_{m w}}{z_{m w}{ }^{2}} T_{i}-\frac{4 \alpha_{m w}}{z_{m w^{2}}} T_{m w}+\frac{2 \alpha_{m w}}{z_{m w}{ }^{2}} T_{o} \\
\frac{d T_{b w}}{d t}=\frac{2 \alpha_{b w}}{z_{b w^{2}}} T_{i}-\frac{4 \alpha_{b w}}{z_{b w}{ }^{2}} T_{b w}+\frac{2 \alpha_{b w}}{z_{b w}{ }^{2}} T_{o} \\
\frac{d T_{s w}}{d t}=\frac{2 \alpha_{s w}}{z_{s w}{ }^{2}} T_{i}-\frac{4 \alpha_{s w}}{z_{s w}{ }^{2}} T_{s w}+\frac{2 \alpha_{s w}}{z_{s w}^{2}} T_{o} \\
\frac{d T_{r}}{d t}=\frac{2 \alpha_{r}}{z_{r}^{2}} T_{i}-\frac{4 \alpha_{r}}{z_{r}^{2}} T_{r}+\frac{2 \alpha_{r}}{z_{r}^{2}} T_{o}
\end{gathered}
$$

$$
\frac{d T_{f}}{d t}=\frac{2 \alpha_{f}}{z_{f}^{2}} T_{i}-\frac{4 \alpha_{f}}{z_{f}^{2}} T_{f}+\frac{2 \alpha_{f}}{z_{f}^{2}} T_{o}
$$

In matrix notation, a system of coupled ordinary differential equations can be concatenated in the deterministic linear state space model in continuous time as given in equation (7). Here, $\mathrm{X}$ denotes the state vector, and $U$ is the input vector. $A$ is the system matrix which characterizes the dynamical behaviour of the system and B is the system input matrix which stipulates how the input signals enter the system.

$$
\frac{d X}{d t}=A X+B U
$$

However, often, equation (7) is not able to exactly predict the future behaviour of the state of the system. An additive noise term is introduced to the model to simulate random variation of the state variables. Introducing a noise term is important because it accounts for:

- modelling approximations such as completely mixed air, ideal gas assumption and lumped states and parameters.

- unrecognized and unmodelled inputs such as wind speed and solar irradiation.

- noise-corrupted measurements.

Then the new model of system dynamics is described by equation 8 and it describes all states in the system. $\omega(\mathrm{t})$ is assumed to be a stochastic process with a zero mean and a given covariance.

$$
d X=A X d t+B U d t+d \omega(t)
$$

Another equation is introduced to represent the measured states of the system which is called the measurement equation and presented in equation (9). The term $\mathrm{v}(\mathrm{t})$ is the measurement error which accounts for the noise affected output signals from different sensors. In this equation, it is assumed that only a linear combination of the states is measured. $\mathrm{C}$ is a constant matrix, which specifies the measured states. D is also a constant matrix, and it accounts for the input variables directly affecting the output.

$$
Y(t)=C X(t)+D U(t)+v(t)
$$

The physical model developed for the single-zone building unit can now be concatenated to obtain a stochastic linear state space model in continuous time corresponding to the equations (8) and (9). We reduced the number of independent parameters by assuming $\alpha_{m w}=\alpha_{b w}=\alpha_{s w}=\alpha_{r}=\alpha_{f}=\alpha$, in order to obtain a simple model for parameter estimation. 
The matrices $\mathrm{X}, \mathrm{A}, \mathrm{B}, \mathrm{U}, \mathrm{Y}$ and $\mathrm{C}$ are given below. The process noise matrix is $\mathrm{W}$ and measurement noise matrix is $\mathrm{V}$.

$$
\begin{aligned}
& X=\left[\begin{array}{llllll}
T_{i} & T_{m w} & T_{b w} & T_{s w} & T_{r} & T_{f}
\end{array}\right]^{T} \\
& A=\left[\begin{array}{cccccc}
\frac{-M_{i} A_{t o t} U_{o}}{\rho_{i} V_{i}\left(M_{i} c_{p_{i}}-R\right)} & 0 & 0 & 0 & 0 & 0 \\
\frac{2 \alpha}{z_{m w}{ }^{2}} & \frac{-4 \alpha}{z_{m w}{ }^{2}} & 0 & 0 & 0 & 0 \\
\frac{2 \alpha}{z_{b w}{ }^{2}} & 0 & \frac{-4 \alpha}{z_{b w}{ }^{2}} & 0 & 0 & 0 \\
\frac{2 \alpha}{z_{\S w^{2}}{ }^{2}} & 0 & 0 & \frac{-4 \alpha}{z_{s w}{ }^{2}} & 0 & 0 \\
\frac{2 \alpha}{z_{r}^{2}} & 0 & 0 & 0 & \frac{-4 \alpha}{z_{r}^{2}} & 0 \\
\frac{2 \alpha}{z_{f}^{2}} & 0 & 0 & 0 & 0 & \frac{-4 \alpha}{z_{f}^{2}}
\end{array}\right] \\
& B=\left[\begin{array}{cc}
\frac{M_{i}}{\rho_{i} V_{i}\left(M_{i} c_{p_{i}}-R\right)} & \frac{M_{i} A_{t o t} U_{o}}{\rho_{i} V_{i}\left(M_{i} c_{p_{i}}-R\right)} \\
0 & \frac{2 \alpha}{z_{m_{w} w^{2}}} \\
0 & \frac{2 \alpha}{z_{b w^{2}}} \\
0 & \frac{2 \alpha}{z_{\tilde{z} w}{ }^{2}} \\
0 & \frac{2 \alpha}{z_{r}^{2}} \\
0 & \frac{2 \alpha}{z_{f}^{2}}
\end{array}\right] \\
& U=\left[\begin{array}{ll}
\dot{Q}_{h} & T_{o}
\end{array}\right]^{T} \\
& Y=\left[\begin{array}{llllll}
y_{1} & y_{2} & y_{3} & y_{4} & y_{5} & y_{6}
\end{array}\right]^{T} \\
& C=\left[\begin{array}{llllll}
1 & 0 & 0 & 0 & 0 & 0 \\
0 & 1 & 0 & 0 & 0 & 0 \\
0 & 0 & 1 & 0 & 0 & 0 \\
0 & 0 & 0 & 1 & 0 & 0 \\
0 & 0 & 0 & 0 & 1 & 0 \\
0 & 0 & 0 & 0 & 0 & 1
\end{array}\right] \\
& W=\left[\begin{array}{llllll}
\omega_{1} & \omega_{2} & \omega_{3} & \omega_{4} & \omega_{5} & \omega_{6}
\end{array}\right]^{T} \\
& V=\left[\begin{array}{llllll}
v_{1} & v_{2} & v_{3} & v_{4} & v_{5} & v_{6}
\end{array}\right]^{T}
\end{aligned}
$$

\section{Parameter estimation}

The goal of the Extended Kalman Filter is to estimate the unmeasured state variables and actual if they are observable, and reduce the noise on the measured process outputs. It involves two step predictor-corrector algorithms. In the first step, the most recent state estimate and an estimation of the error covariance are presented. These predictions are used to estimate othe state variables at the current time. In the second step, the predicted state estimate in the first step is corrected to generate an updated state estimate by incorporating the latest process measurements. For a detailed description of the Kalman Filter, please consult Simon (2006). The unknown parameters of a the model are estimated using the Extended Kalman Filter (EKF) by augmenting the state with the parameters. This process may transform a linear system of equations to a non-linear system. Since EKF can equally be applied to linear and non-linear systems, the parameterization problem can be solved depending on the system observability. Implementation steps of the discrete time EKF are summarized in Table 1.

\subsection{Structural observability analysis}

Observability of a dynamic system determines how well the states can be inferred from input-output data. The concept of observability characterizes whether a given set of measurements is adequate to estimate the state of the system [Simon (2006)]. Structural observability analyzes the observability of a system based on the system structure. It gives a necessary condition for observability which means that if a system is not structurally observable, then it is not observable [Perera et al. (2015)]. A method for verifying the structural observability of a dynamic system is presented in detail in Perera et al. (2015) using graph-theoretic techniques. It offers a visual means to pinpoint measurements needed to estimate state, disturbances and parameters or to detect which cannot be estimated at all in the augmented system. When estimating the unknown parameters of a system, Kalman filtering can be used by augmenting the system with them as state variables. This necessitates a check up of the observability of the augmented system.

To analyze the structural observability, a digraph of the augmented system was created first. Definitions of some important terms given in Perera et al. (2015) that are used to interpret the graph are given below to assist the reader to understand the theory behind structural observability.

- Nodes of the graph represents augmented states, inputs, and output variables.

- An edge connects two nodes in the system. In a directed graph (digraph) nodes are connected with directed lines.

- A path has an initial node and a final node. The number of edges in a path is called the length of it. A Path can be a simple/elementary path (path contains no node appearing more than once) or a closed path (path with initial and final nodes are identical). In our system, no closed paths are observed.

- If the closed path has no node appearing more than once except the initial and final nodes, then it is a cycle. Cycles having a length of one, are 
1. System equation: $x_{k}=f_{k-1}\left(x_{k-1}, u_{k-1}, \omega_{k-1}\right)$ and measurement equation: $y_{k}=h_{k}\left(x_{k}, \omega_{k}\right)$ are known.

2. Specify the process noise matrix $Q_{k-1}$, and measurement noise matrix $R_{k-1}$.

3. Initialize the filter with state estimates $\hat{x}_{k-1}^{+}$, and state estimation covariance $\hat{P}_{k-1}^{+}$.

4. Compute the partial derivative matrices $F_{k-1}=\left.\frac{\partial f_{k-1}}{\partial x}\right|_{\hat{x}_{k-1}^{+}}$and $L_{k-1}=\left.\frac{\partial f_{k-1}}{\partial \omega}\right|_{\hat{x}_{k-1}^{+}}$.

5. Perform the time update of the state estimate $\hat{x}_{k}^{-}=f_{k-1}\left(\hat{x}_{k-1}^{+}, u_{k-1}, 0\right)$, and estimation error covariance $P_{k}{ }^{-}=F_{k-1} P_{k-1} F_{k-1}^{T}+L_{k-1} Q_{k-1} L_{k-1}^{T}$.

6. Compute the partial derivative matrices $H_{k}=\left.\frac{\partial h_{k}}{\partial x}\right|_{\hat{x}_{k}^{-}}$and $M_{k}=\left.\frac{\partial h_{k}}{\partial v}\right|_{\hat{x}_{k}^{-}}$.

7. Compute the Kalman filter gain matrix $K_{k}=P_{k}^{-} H_{k}^{T}\left(H_{k} P_{k}^{-} H_{k}^{T}+M_{k} R_{k} M_{k}^{T}\right)^{-1}$.

8. Perform the measurement update of the state estimate $\hat{x}_{k}^{+}=\hat{x}_{k}^{-}+K_{k}\left[y_{k}-h_{k}\left(\hat{x}_{k}^{-}, 0\right)\right]$, and estimation error covariance $P_{k}=\left(I-K_{k} H_{k}\right) P_{k}$.

9. To implement EKF, the matrix $F_{k-1}$ should be invertible and the pair $F_{k-1}$ and $H_{k-1}$ should be observable.

called self-cycles or loops. In the system, six loops can be observed around each state variable.

- A directed cactus (figure 3) is made out of a stem and buds connected in a special way.

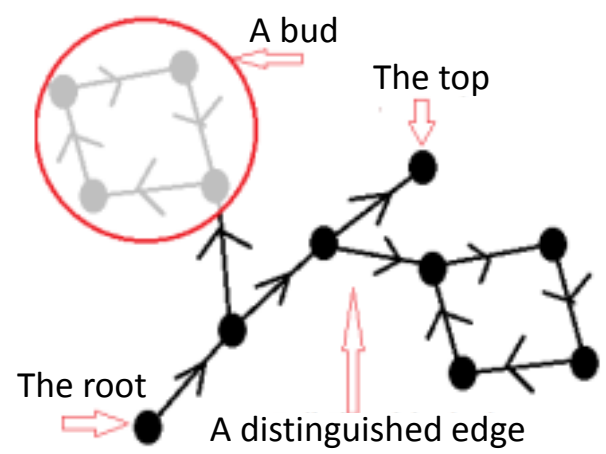

Figure 3: A cactus with two buds. The initial node and final node of the stem is called root and top [Perera et al. (2015)].

The digraph of the augmented system is presented in figure 4 . The system is said to be structurally observable if and only if cacti ${ }^{1}$ span the digraph as described by Perera et al. (2015). That is for the model to be structurally observable, the graph with root node must be directed in the forward path and terminates

\footnotetext{
${ }^{1}$ The plural of "cactus" is "cacti"
}

at the final node. If the graph contains any buds, then these buds need to be in the cycle. The initial node should be output measurements, and the final node can be unknown parameters, disturbances or outputs. To check the structural observability of the given building heating model, each cactus from the digraph presented in figure 4 needs to be analyzed. In this case, cactus 1 starts with output measurement $y_{1}$ directed in the forward direction path and connected to the state $T_{i}$. Then $T_{i}$ is joined with the unknown parameter $U_{o}$. Cactus 2 starts with the measurement $y_{5}$ and directed in the forward path to connect with state $T_{r}$. Finally $T_{r}$ directs towards the unknown parameter $\alpha$. Several other cacti can be observed from the figure connecting $\alpha$, such as $y_{2} \rightarrow T_{m w} \rightarrow \alpha, y_{3} \rightarrow T_{b w} \rightarrow \alpha, y_{4} \rightarrow T_{s w}$ $\rightarrow \alpha$ and $y_{6} \rightarrow T_{f} \rightarrow \alpha$.

Based on the observations, root nodes of the graph are connected to the final nodes in a forward path, and there are no buds in the system. Therefore, the model is structurally observable and satisfies the primary condition to be observable. The observability matrix which is constructed using system matrix $\mathrm{A}$ and constant matrix $\mathrm{C}$ has full rank. Hence, the system is observable, and the unknown parameters can be estimated based on the output measurements.

The figure 5 is presented to illustrate the idea behind the structurally un-observable systems. Consider the same building heating model having individual overall heat transfer coefficients and individual thermal diffusivities to characterize the heat flow through walls, 


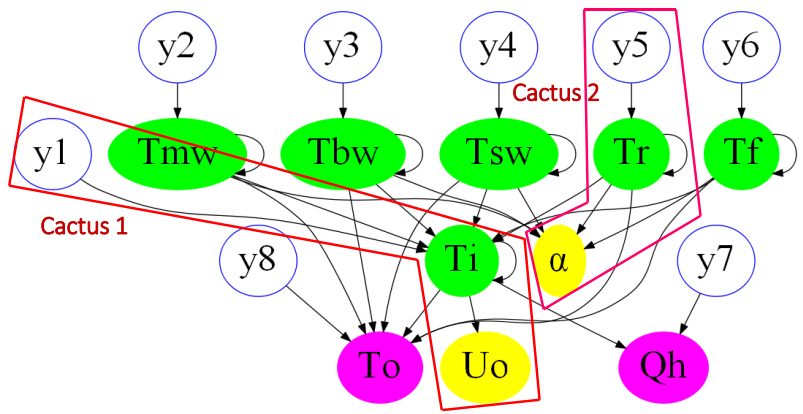

Figure 4: Digraph of the augmented system.

roof and floor. The digraph is developed for the new scenario to analyze the model structure. In this case, cactus 3 starts with output measurement $y_{1}$ and directed in the forward direction path to state $T_{i} . T_{i}$ is connected with the five unknown parameters $U_{m w}$, $U_{b w}, U_{s w}, U_{r}$ and $U_{f}$. Hence, cactus 3 has five unknown augmented states as final nodes which makes the new model structurally not observable. Therefore, the new model does not satisfy the necessary condition to be observable, and the individual parameters cannot be estimated using the Extended Kalman filter.

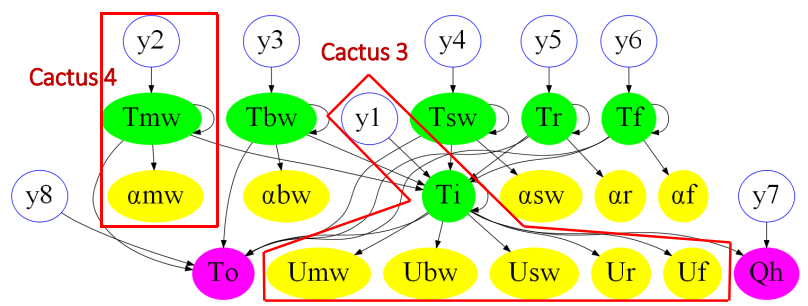

Figure 5: Digraph of the system with individual overall heat transfer coefficients and individual thermal diffiusivities.

\section{Results}

The presented model is implemented in the Extended Kalman Filter algorithm to estimate the two unknown parameters $\mathrm{U}_{0}$ and $\alpha$. An off-line approach is used for the identification. The properties $\rho_{i}$ and $M_{i}$ are assumed to vary depending on the state variable (indoor temperature) and, $c_{p_{i}}$ is considered as a constant throughout the model execution. However, it will not make a considerable effect on the results if the properties are assumed to be constant because they are related to air and do not have a high variability based on the temperature.

Two data sets were used describing the cooling and
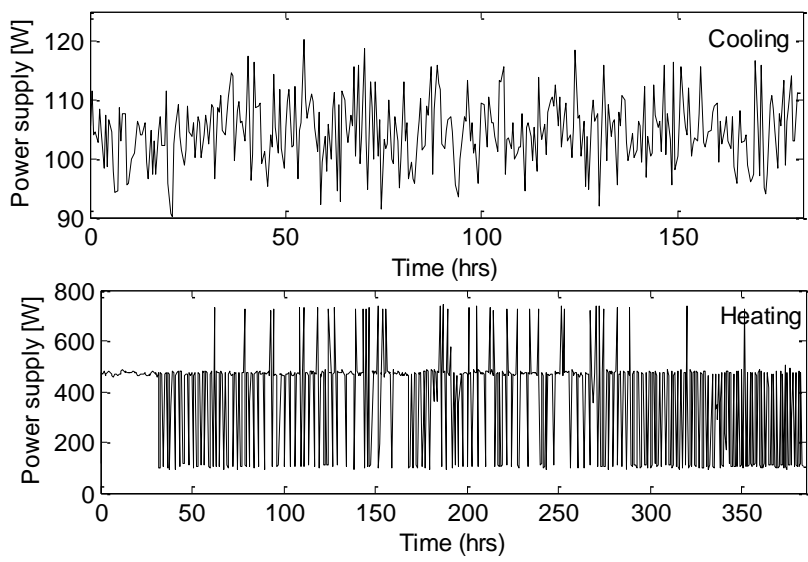

Figure 6: Total power supply to the building during cooling and heating $[\mathrm{W}]$.
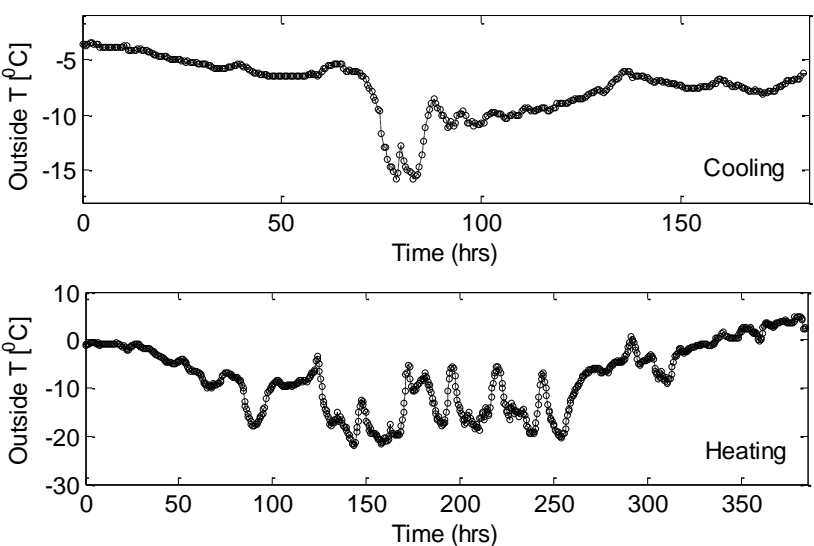

Figure 7: Outside temperature variation at the location of the test building $\left[{ }^{0} \mathrm{C}\right]$. 
heating of the test building. The cooling data set was recorded from 02-01-2016 to 10-01-2016, and the heating data set was recorded from 11-01-2016 to 27-012016. Each data set consists of 362 and 769 samples respectively with 0.5-hour sampling interval. Data used for external conditions such as outside temperature are also collected at the building location. Input power consumption from the on-off controlled heater is also measured. The quality of the parameterization largely depends on the quality of the data used for identification. The collected data ensures the excitation of most of the system conditions depending on the climate of the building location. Power consumption representing the system input and the outside temperatures describing the system disturbance are illustrated in figure 6 and figure 7 .

The power consumption of the building depends on a heater and a computer. The computer is placed inside for data logging. The low limit of the power consumption is approximately $95-100 \mathrm{~W}$, which is the power used by the computer. During cooling the heater is completely off and the average power consumption of the computer is $105 \mathrm{~W}$. According to the heater dynamics seen in figure 6 during heating, the heater is automatically turned on and off several times during the experimental period. The outdoor temperatures obtained for the two cases show a significant variability throughout the period.
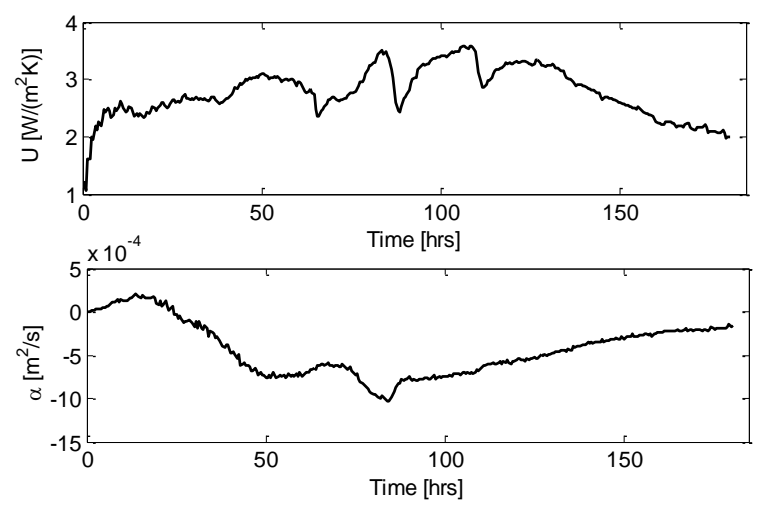

Figure 8: Estimated value of the overall heat transfer coefficient, $\mathrm{U}_{0}$ and thermal diffusivity, $\alpha$ using EKF for cooling.

The EKF and the system model manage to tune the parameters and lead to a very good temperature tracking for the six state variables (inside, three walls, roof, and floor temperatures). Figure 8 and figure 9 show the evolution of the two parameters $\left(\mathrm{U}_{0}\right.$ and $\alpha$ ) over time for both cooling and heating. For the cooling experiment, the parameters do not reach stable values
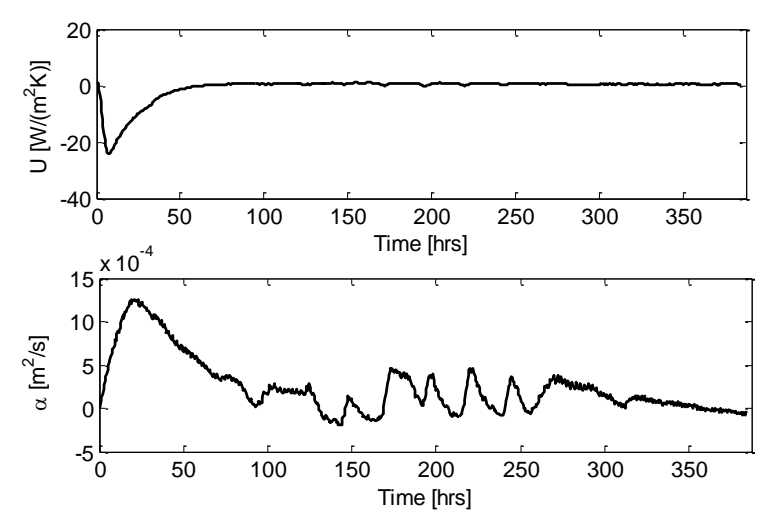

Figure 9: Estimated value of the overall heat transfer coefficient, $\mathrm{U}_{0}$ and thermal diffusivity, $\alpha$ using EKF for heatling.

during the experimental period. However, during heating, the overall heat transfer coefficient reaches steady state while thermal diffusivity does not. Both parameters take negative values at some points which is not physically interpretable. Therefore, they rather seem to follow the building dynamics based on the inputs and disturbances.

The predicted and measured values of each state variable for both cooling, and heating are illustrated in figures 10 and 11 respectively.

\section{Discussion}

The model is found to be stable and observable for all the experiments. The state estimations follow the measurements closely. However, the estimated parameters do not converge to a single value but reflect the heat dynamics of the building. One main limitation associated with this method is the loss of physical interpretability of the parameters. Both estimated parameters take negative values at some points which do not have physical meaning. Also, it is challenging to obtain real values for the parameters because they depend on the thermal properties of the construction materials, inputs to the system and disturbances. The thermal diffusivity of each component can be computed theoretically if thermal conductivity, density and specific heat capacity of each material are known.

There are several disturbances present in the system that have not been modelled. The EKF can be improved by measuring the contribution of each disturbance such as solar irradiation and wind speed. However, this will increase the model complexity and hence result in high simulation times. Therefore, there is a 

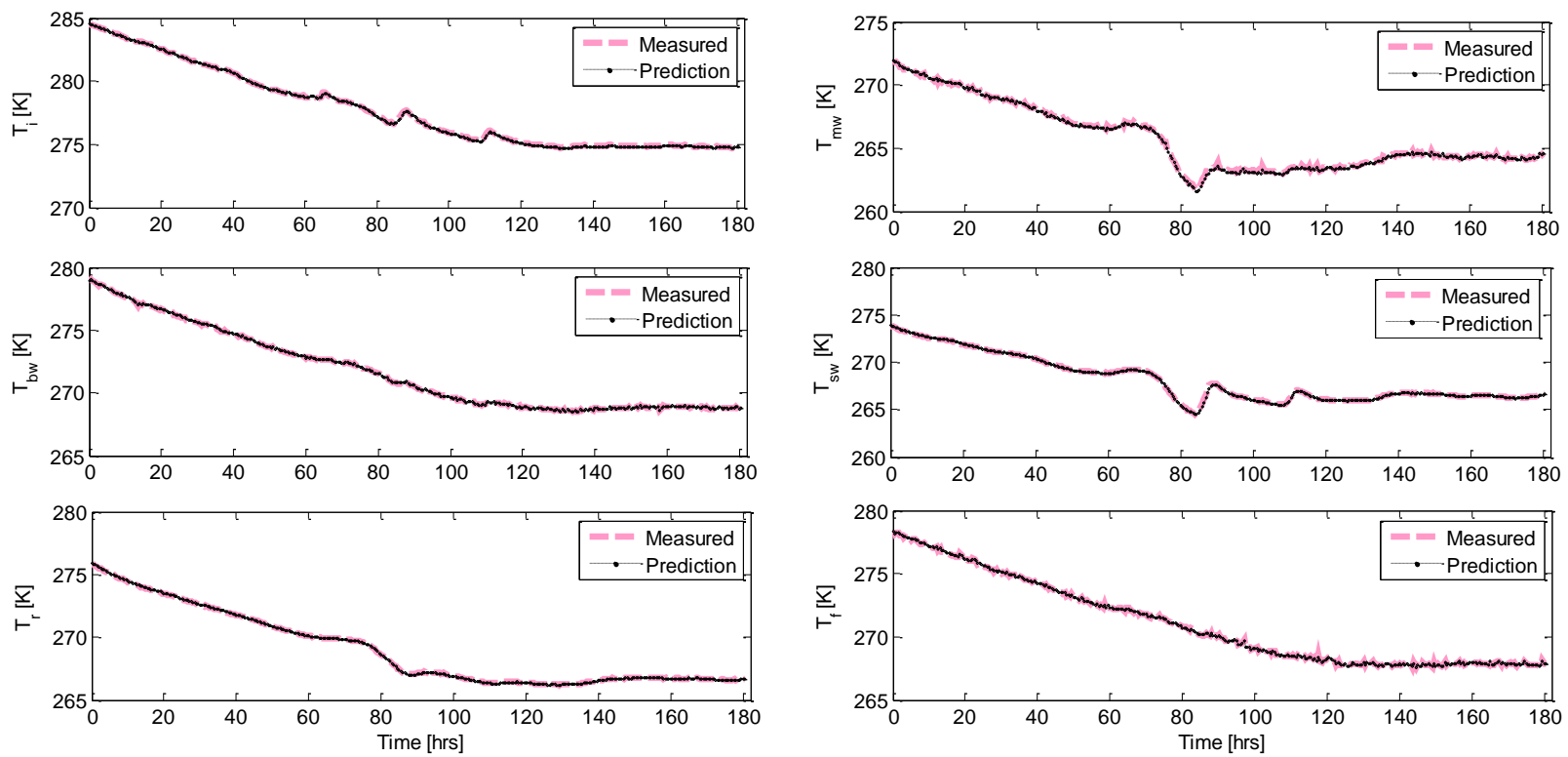

Figure 10: Measured and predicted temperatures of the six state variables using EKF for cooling.
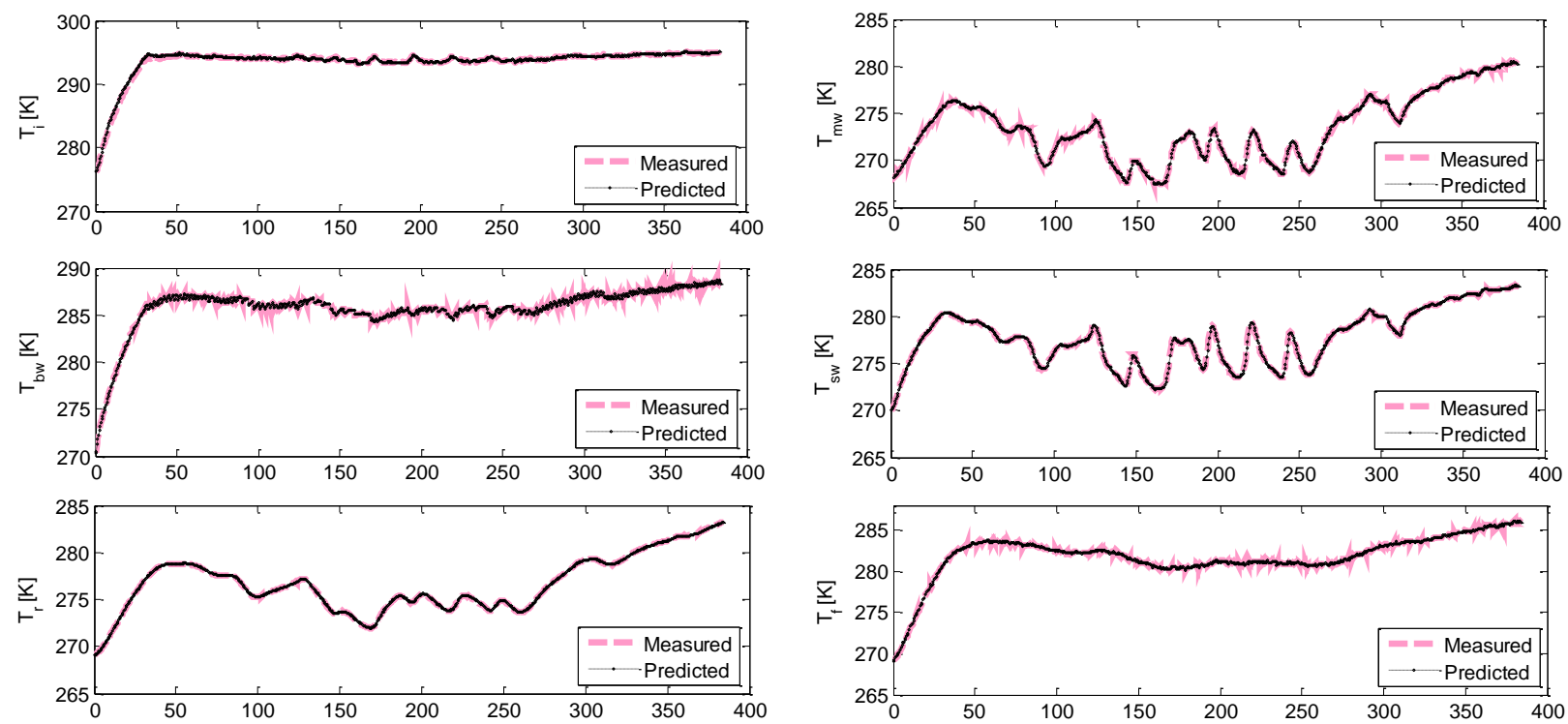

Figure 11: Measured and predicted temperatures of the six state variables using EKF for heating. 
trade-off between the system complexity and required precision.

The computational time of the system is around 1 hour for the simulated period using a laptop computer with a CORE i5 processor having Windows 7 operating system and 8 GB memory. For one day period, the simulation time is around 2 minutes and hence the computational time seems acceptable. The EKF estimator has relatively less number of state variables and comparatively less computational time that makes it worthwhile to implement an on-line controller with online parameter estimation for the mentioned building.

\section{Conclusion}

Advanced control algorithms require a mathematical building heating model to tailor the controller towards building dynamics. As buildings are complex and nonlinear dynamic systems, the development of the precise and reliable building heating model is a challenging task. However, non-linear and reliable models tend to be computationally intensive for the application of on-line building control systems. A dynamic model is developed for the specified single-zone building which consists of six state variables, one input, one disturbance and two unknown parameters. The state variables are augmented with the two unknown parameters which transform the model to a non-linear system. The suggested model is declared to be sufficient to capture the essential dynamics of the building. Procedure for estimation of unknown model parameters in the developed continuous time model based on the measured building performance data in discrete time is presented in this report. The proposed methodology is based on the Extended Kalman Filter, which requires the examination of the observability of the system. The observability of the model is interpreted using structural observability analysis using graph theory. The application of the EKF algorithm to the system estimates the unknown parameters of the model. The EKF is found to be a promising algorithm as it yields accurate indoor temperature estimations as well as wall, roof and floor temperatures. However, predicted parameres lose their physical interpretability and can result in negative value estimations. This can probably be caused by unmodelled dynamics or process disturbances Computational time of the proposed model with EKF is low and thus suitable for real-time implementation.

\section{Future work}

The parameters of the model are estimated using an off-line approach. A framework for on-line estimation of unknown parameters of the building needs to be studied. On-line approach concurrently tunes the parameters of the model and provides future state estimates based on the predicted inputs and disturbances. Such strategies are important for the on-line control in Building Energy Management Systems. Further, other techniques such as Unscented Kalman Filter, Ensemble Kalman Filter and Particle Filters can be applied to check their performance in parameter estimation.

\section{Author Contributions}

D. Wathsala U. Perera designed the experiments, performed the analysis and was responsible for preparing the manuscript. M. Anushka S. Perera provided the theoretical information for conducting the structural observability analysis for the building heating model and implemented the Extended Kalman Filter algorithm. Carlos F. Pfeiffer and Nils-Olav Skeie provided ideas to analyse the results and revised the manuscript in all versions.

\section{References}

Bacher, P. and Madsen, H. Identifying suitable models for the heat dynamics of buildings. Energy and Buildings, 2011. 43(7):1511-1522. doi:10.1016/j.enbuild.2011.02.005.

Dounis, A. and Caraiscos, C. Advanced control systems engineering for energy and comfort management in a building environment-a review. Renewable and Sustainable Energy Reviews, 2009. 13(6-7):12461261. doi:10.1016/j.rser.2008.09.015.

Fux, S. F., Ashouri, A., Benz, M. J., and Guzzella, L. Ekf based self-adaptive thermal model for a passive house. Energy and Buildings, 2014. 68, Part C:811817. doi:10.1016/j.enbuild.2012.06.016.

Kristensen, N., Madsen, H., and Jrgensen, S. Parameter estimation in stochastic grey-box models. Automatica, 2004. 40(2):225-237. doi:10.1016/j.automatica.2003.10.001.

Maasoumy, M., Moridian, B., Razmara, M., Shahbakhti, M., and Sangiovanni-Vincentelli, A. Online simultaneous state estimation and parameter adaptation for building predictive control. In $A S M E$ 2013 Dynamic Systems and Control Conference2013. 2013. doi:10.1115/DSCC2013-4064.

Madsen, H. and Holst, J. Estimation of continuoustime models for the heat dynamics of a building. Energy and buildings, 1995. 22(1):67-79. doi:10.1016/0378-7788(94)00904-X. 
Table 2: Nomenclature

\begin{tabular}{llll}
\hline Symbols & \multicolumn{2}{l}{ Subscripts } \\
$\mathrm{A}$ & Surface area $\left[\mathrm{m}^{2}\right]$ & $i$ & Outside \\
$\mathrm{c}_{p}$ & Specific heat capacity $[\mathrm{J} /(\mathrm{kg} . \mathrm{K})]$ & $b w$ & Sustainable wall \\
$\mathrm{M}$ & Molar mass $[\mathrm{kg} / \mathrm{mol}]$ & $f$ & Floor \\
$\mathrm{R}$ & Gas constant $[\mathrm{J} /(\mathrm{mol} . \mathrm{K})]$ & $m w$ & Solid wall \\
$\dot{Q}_{h}$ & Heat supply $[\mathrm{W}]$ & $o$ & Outside \\
$\mathrm{T}$ & Temperature $[\mathrm{K}]$ & $s w$ & Standard wall \\
$\mathrm{t}$ & Time $[\mathrm{s}]$ & tot & Total \\
$\mathrm{U}$ & Overall heat transfer coefficient & & \\
$\mathrm{V}$ & Volume $\left[\mathrm{m}^{3}\right]$ & & \\
$\mathrm{z}$ & Thickness $[\mathrm{m}]$ & & \\
$\alpha$ & Thermal diffusivity $\left[\mathrm{m}^{2} / \mathrm{K}\right]$ & & \\
$\rho$ & density $\left[\mathrm{kg} / \mathrm{m}^{3}\right]$ & & \\
\hline
\end{tabular}

Martincevic, A., Starcic, A., and Vasak, M. Parameter estimation for low-order models of complex buildings. In Innovative Smart Grid Technologies Conference Europe (ISGT-Europe), 2014 IEEE PES. 2014. doi:10.1109/ISGTEurope.2014.7028767.

Perera, D. W. U., Pfeiffer, C., and Skeie, N.-O. Modelling the heat dynamics of a residential building unit: Application to norwegian buildings. Modeling, Identification and Control, 2014a. 35(1):43-57. doi:10.4173/mic.2014.1.4.

Perera, M. A. S., Lie, B., and Pfeiffer, C. F. Structural observability analysis of large scale systems using modelica and python. Modeling Identification and Control, 2015. 36(1):53-65. doi:10.4173/mic.2015.1.4.

Perera, W., Pfeiffer, C. F., and Skeie, N.-O. Modeling and simulation of multi-zone buildings for bet- ter control. In 55th Conference on Simulation and Modelling, Aalborg, Denmark: Linkping University Electronic Press. 2014b.

Perez-Lombard, L., Ortiz, J., and Pout, C. A review on buildings energy consumption information. Energy and Buildings, 2008. 40:394-398.

Radecki, P. and Hencey, B. Online building thermal parameter estimation via unscented kalman filtering. In American Control Conference (ACC). 2012. doi:10.1109/ACC.2012.6315699.

Simon, D. Optimal state estimation : Kalman, H Infinity and nonlinear approaches. Wiley-Interscience, 2006.

Walker, D. M. System identification using constrained kalman filters. In International Symposium on Nonlinear Theory and its Applications. 2005. 